\title{
УДК: 159.9.072.432 - 616.31-089-616.31-06
}

\section{МЕДИКО-ПСИХОЛОГИЧЕСКИЕ ОСОБЕННОСТИ ВЕДЕНИЯ ПАЦИЕНТОВ С РАЗЛИЧНЫМИ ФОРМАМИ САХАРНОГО ДИАБЕТА В АМБУЛАТОРНЫХ УСЛОВИЯХ МАУ “СП № 12»}

\author{
Петров И.А. ${ }^{1}$, Чернавский А.Ф., ${ }^{1,2}$ Бадалян А.А. ${ }^{1}$, Огнев М.Ю. ${ }^{1}$
}

1 Муниципальное автономное учреждение «Стоматологическая поликлиника № 12», г. Екатеринбург, Российская Федерация

2 ФГАОУ ВО «УрФУ имени первого Президента России Б.Н. Ельцина», г. Екатеринбург, Российская Федерация

\section{Аннотация}

Предмет. Нарушение психоэмоционального состояния и ожидание болевого синдрома при проведении стоматологических вмешательств ведет к формированию стрессовых реакций организма, дезорганизует работу функциональных систем организма и, следовательно, ведет к развитию негативных, влияющих на жизнь и здоровье пациентов стоматологического профиля функциональных изменений. Основной задачей врачи стоматологи и клинические психологии в настоящее время считают преодоление эмоционального стресса, особенно у пациентов с такой общесоматической патологией, как эндокринная.

Цель. Разработать новую стратегию к ведению пациентов с различными формами сахарного диабета в условиях амбулаторно-поликлинического приема.

Методология. Использование психологических тестов, опросников, динамического наблюдения за психофизиологическими и медицинскими показателями пациентов стоматологического профиля с сахарным диабетом, методы статистической обработки полученных данных.

Результаты проведенного нами исследования показывают, что 98,5\% всех испытуемых испытывали выраженное эмоциональное напряжение перед стоматологическим вмешательством. В контрольной группе (без эндокринной патологии) страх перед стоматологическим вмешательством испытывали $61 \%$ пациентов, в группе пациентов, больных сахарным диабетом I типа, - 92\% человек, в группе с сахарным диабетом II типа - 79\%. 39\% всех испытуемых после стоматологического вмешательства отметили, что ожидание боли или манипуляции стоматолога ведут к возникновению эмоционального стресса, порой к неадекватному поведению и недоверию к действиям и рекомендациям врача-стоматолога.

Выводы. Результаты исследования имеют практическое значение при работе с пациентами, страдающими сахарным диабетом. Врачам стоматологам-хирургам при планировании стоматологического вмешательства необходимо учитывать тип сахарного диабета, влияющего на лабильность настроения. Наличие у пациентов раздражительности, мнительности, тревожности, подавленности, угнетенности, общей слабости, адинамии, повышенной утомляемости, вялости, сонливости, снижение работоспособности, эмоциональной неустойчивости, повышенной конфликтности, хронических, довольно выраженных личностных изменений, обусловленных заболеванием, - прямое показание для обращения к клиническому психологу и проведения психотерапевтической подготовки перед стоматологическим вмешательством.

Ключевые слова: эндокринная патология, сахарный диабет, страх перед стоматологическим вмешательством.

\section{Признательность}

Авторы выражают благодарность стоматологическому факультету УГМУ за организационно-методическую поддержку исследований практического здравоохранения.

\begin{tabular}{|c|c|}
\hline Адрес для переписки: & Correspondence address: \\
\hline $\begin{array}{l}\text { Александр Фридрихович Чернавский } \\
\text { к.пс.н., доиент кафедры клинической психологии и } \\
\text { психофизиологии ИСПН ФГАОУ ВО «УрФУ имени первого } \\
\text { Президента России Б.Н. Ельцина», Екатеринбург, Российская } \\
\text { Федерация } \\
\text { dосепt.alех@таil.ru } \\
\text { Aдресдля переписки: 620075, г. Екатеринбург, ул. Техническая, д. } 28 \\
\text { Тел. +7(922) 106-26-98 }\end{array}$ & $\begin{array}{l}\text { Aleksandr F. CHERNAVSKY } \\
\text { PhD in psychological sciences, Associate Professor, Department } \\
\text { of Clinical Psychology and Psychophysiology, Institute } \\
\text { of Social and Political Sciences, Ural Federal University, } \\
\text { Ekaterinburg, Russia } \\
\text { docent.alex@mail.ru } \\
\text { 620075, Ekaterinburg, Tehnicheskaya, } 28 \\
\text { Tel.: +7 (922) 106-26-98 }\end{array}$ \\
\hline Образец цитирования: & For citation: \\
\hline $\begin{array}{l}\text { Петров И.А., Чернавский А.Ф., Бадалян А.А., Огнев М.Ю. } \\
\text { «Медико-психологические особенности ведения пациентов } \\
\text { с различными формами сахарного диабета в амбулаторных } \\
\text { условиях МАУ «СП № } 12 » » \\
\text { Проблемы стоматологии, 2017, Т. 13, № 1. С. 114-120 } \\
\text { dоі: } 10.18481 / 2077-7566-2017-13-1-114-120 \\
\text { () Петрова И.А. и соавт., } 2017\end{array}$ & $\begin{array}{l}\text { Petrov I.A., Chernavsky A.F., Badalyan A.A., Ognev M.Yu. } \\
\text { «Medico-psychological features of case management of } \\
\text { patients with various forms of diabetes mellitus provided with } \\
\text { ambulatory care in the municipal autonomous institution } \\
\text { «dental clinic №12»» } \\
\text { The actual problems in dentistry, } \\
\text { 2017. Vol. 13, № 1, pp. 114-120 } \\
\text { DOI: } 10.18481 / 2077-7566-2017-13-1-114-120\end{array}$ \\
\hline
\end{tabular}




\title{
MEDICO-PSYCHOLOGICAL FEATURES OF CASE MANAGEMENT OF PATIENTS WITH VARIOUS FORMS OF DIABETES MELLITUS PROVIDED WITH AMBULATORY CARE IN THE MUNICIPAL AUTONOMOUS INSTITUTION «DENTAL CLINIC №12»
}

\author{
Petrov I.A. ${ }^{1}$, Chernavsky A.F. ${ }^{1,2}$, Badalyan A.A. ${ }^{1}$, Ognev M.Yu. ${ }^{1}$ \\ Municipal autonomous institution «Dental clinic № 12», Ekaterinburg, Russia \\ Ural Federal University, Ekaterinburg, Russia
}

\begin{abstract}
Background Disturbance of a patient's psycho-emotional state, when he or she anticipates pain during dental interventions, causes the organism's stress reactions, disorganizes its functional systems and, therefore, leads to the development of negative functional changes. Such changes might affect dentistry patients' life and health. Today, dentists and clinical psychologists believe it to be their duty to help patients to overcome the emotional stress, which problem particularly concerns people with such a general somatic pathology as the endocrine one.

Objectives Development of a new strategy for the case management of patients with various forms of diabetes in the outpatient reception conditions.

Methods The use of psychological tests; questionnaires; dynamic monitoring of psycho-physiological and medical indices of patients of dental profile with diabetes; statistical processing methods of the obtained data.

Results The results show that $98.5 \%$ of all the tested patients experienced significant emotional stress prior to a dental treatment. In the control group (without endocrine pathology), 61\% of the patients experienced dental fear, with this number being $92 \%$ in the group of the patients suffering from diabetes mellitus type 1 , and $79 \%$ in the group suffering from diabetes type $2.39 \%$ of all the patients questioned after dental interventions noted that the anticipation of pain or dentist manipulations was leading to subsequent emotional stress, sometimes expressed in inappropriate behavior and mistrust to the actions and recommendations of the dentist.

Conclusions The results obtained have practical importance for patients suffering from diabetes. When planning dental interventions in such patients, dentists and surgeons should take into account patients' type of diabetes affecting their mood lability. In case a patient reveals such symptoms as irritability, suspiciousness, anxiety, sadness, suppression, general weakness, adinamia, fatigue, atony, sleepiness, decreased performance capablity, emotional volatility, increased proneness to conflict, presence of chronic, rather pronounced personality changes arising from illness, this should be treated as a direct indication for consulting a clinical psychologist and carrying out a psychological preparation prior to further dental interventions.
\end{abstract}

Keywords: endocrine pathology, diabetes mellitus, dental fear

\section{Acknowledgments}

The authors would like to express their gratitude to the Dental Faculty of USMU for organizational and methodological support of this practical health care research.

\section{Введение}

На сегодняшний день выходит на первый план психосоциальная проблема ведения пациентов с сахарным диабетом. Исследования, проводимые в нашей стране и за рубежом, в частности в Германии, доказывают, что у 84-94\% пациентов перед стоматологическим вмешательством возникают негативные психосоматические реакции, начинающиеся с напряжения, неясной тревоги, проявлений страха в виде преддействия (психофизиологические изменения в организме, ощущаемые на подсознательном уровне), а порой и возникновение фобических реакций [9]. Ряд исследователей в своих работах отмечают, что пациенты с сахарным диабетом наиболее подвержены психоэмоциональному напряжению, что существенно влияет на повышение болевой чувствительности, и перед проведением стоматологических вмешательств для предупреждения развития негативных реакций психофизиологического характера необходим не только правильный выбор метода премедикации и местного обезболивания, но и психотерапевтическая подготовка. Оказание стоматоло- гической помощи пациентам с сахарным диабетом является одной из актуальных проблем [7, 8, 10].

\section{Актуальность}

В последние 30 лет отмечается резкий рост распространенности и заболеваемости сахарным диабетом, особенно в промышленно развитых странах, где на долю этого заболевания приходится 5-6\% населения, и эта цифра имеет тенденцию к увеличению, что представляет существенную угрозу для жизни и здоровья трудоспособного населения в большинстве индустриально развитых стран и в Российской Федерации [1].

Распространенность диабета в России составляет 3,5\%, ежегодный прирост в конце тысячелетия составляет 5\%. Каждые 10-15 лет число больных сахарным диабетом удваивается [2]. Это происходит в основном за счет прироста больных, страдающих сахарным диабетом второго типа. У пациентов с сахарным диабетом отмечается высокая заболеваемость и интенсивность поражения твердых тканей зубов и пародонта и почти $100 \%$ нуждаемость в стоматологическом лечении [1]. 
Все чаще встречаются пациенты с эндокринной патологией и на стоматологическом приеме. Социальная среда не формирует в настоящее время у пациентов подобного профиля выраженной мотивации на сохранение стоматологического здоровья, и, как показывает статистика, порядка $58 \%$ пациентов с эндокринной патологией становятся посетителями хирургического отделения стоматологической поликлиники. Стоматологический прием пациентов с сахарным диабетом имеет свои особенности, которые касаются как организации приема, так и особенностей медицинского наблюдения и лечения лиц, имеющих данную эндокринную патологию. Физиология заживления лунки после операции удаления зуба у данной категории пациентов достаточно изучена и во многом зависит от ангиогенеза, состояния местного иммунитета. Наши наблюдения подтверждают, что нарушение кровообращения, повышенная гликемия часто вызывает у таких пациентов выраженные местные проявления, проявляющиеся как сухость в слизистой полости рта, снижение секреторной функции слюнных желез, трещины на губах. В полости рта пациента нарушается микробный баланс, увеличивается количество условно патогенной микрофлоры. При этом идет разрушение структуры зубной эмали и развивается кариес. Защитные функции в организме больного сахарным диабетом значительно снижаются, в результате чего повышается риск инфицирования операционных ран в полости рта и развитие серьезных осложнений челюстно-лицевой области (остеомиелит лунки, абсцесс, флегмона и другие) [4]. Операция удаления зубов у пациента, страдающего сахарным диабетом, без проведения специальной подготовки может вызвать острый воспалительный процесс и даже декомпенсацию болезни.

К сожалению, еще достаточное количество врачей стоматологов не учитывают психоэмоциональное и, как следствие, психофизиологическое состояние пациентов перед стоматологическим вмешательством. Наличие страха перед стоматологическим вмешательством приводит к проявлению психологического защитного механизма, приводящего к усугублению стоматологического заболевания. На многие стоматологические вмешательства, требующие инвазивных вмешательств для их лечения, пациенты смотрят как на серьезные и угрожающие их жизни, поэтому часто сопротивляются попыткам врача стоматолога осуществить необходимый объем лечения $[3,10]$.

Основным психопатологическим синдромом у пациентов, страдающих сахарным диабетом, является астенический, доминирующие симптомы этого состояния - повышенная ранимость, обидчивость, слезливость, быстрая утомляемость, раздражительность, колебание настроения (от глубокого недовольства собой, вялости до состояния эйфории, экзальтации с переоценкой своих способностей, многоречивостью, пренебрежительным отношением к себе и лечению)
[5]. Проведенное нами психологическое наблюдение за пациентами подтвердило наличие синдромов астенического ряда у лиц, страдающих сахарным диабетом. Проявление синдромов связано с периодами сахарного диабета, отражающими прогредиентный характер заболевания. Начальная стадия заболевания проявляется астено-невротическим и собственно астеническим синдромами, при прогрессировании заболевания на первое место выходит астено-дистемический синдром, а заключительная стадия заболевания характеризуется снижением интеллекта, обеднением психики, наблюдаются астено-апатический и астеноадинамический синдромы.

Наши наблюдения показывают, что у пациентов с сопутствующей патологией - сахарный диабет, получавших хирургическое лечение в нашей поликлинике, под воздействием средовых факторов чаще всего проявляется астено-невротический синдром, характеризующийся следующими симптомами:

- выраженная эмоциональная лабильность, с готовностью к реакциям раздражения по любому поводу;

- утомляемость, усталость ощущается к концу рабочего дня;

- головные боли нестойкого характера;

- изменения памяти, повышенная забывчивость, связанная с рассеянным вниманием.

Все вышеизложенное обусловило актуальность и необходимость проведения настоящего исследования, определило его цель и задачи.

\section{Цель исследования}

Выработать адекватные подходы к ведению пациентов с различными формами сахарного диабета в условиях амбулаторно-поликлинического приема.

\section{Задачи исследования}

1. Изучение основных особенностей проведения операции удаления зуба у пациентов с сахарным диабетом;

2. Определение организационных особенностей приема пациентов с сахарным диабетом;

3. Изучение психологических аспектов работы с пациентами, страдающими эндокринной патологией - сахарным диабетом.

\section{Практическая значимость работы обусловлена:}

1. Высокой нуждаемостью больных сахарным диабетом в стоматологической помощи;

2. Выявлением особенностей психоэмоционального состояния и динамики физиологических показателей у пациентов с сахарным диабетом 1 и 2 типа на этапе хирургического стоматологического лечения.

3. Необходимостью разработки рекомендаций по оказанию психологической помощи на амбулаторном стоматологическом приеме пациентов с сахарным диабетом в зависимости от тяжести заболевания. 


\section{Материалы и методы исследования}

За период с января 2015 по сентябрь 2016 года в хирургическое отделение муниципального автономного учреждения «Стоматологическая поликлиника № 12» обратилось 7550 пациентов. Из них 5200 для операции удаления зуба: по поводу хронического пародонтита 1300 , осложненного кариеса - 3900. Возраст пациентов - от 18 до 82 лет. Проведена выборка, основанная на данных анамнеза жизни и заболевания, а также анкеты здоровья, выделены три группы пациентов, с учетом типа сахарного диабета, без деления по возрасту, всего 420 человек. После изучения полученных данных сформированы три группы общей численностью 360 человек:

1) пациенты с сахарным диабетом I типа - 30;

2) пациенты с сахарным диабетом II типа - 150;

3) контрольная группа - пациенты без соматической патологии -180 .

При сборе материалов в выделенной группе перед проведением стоматологического лечения проводились методы психологического исследования: тест, опрос, беседа. По специально разработанным тестам оценивалось психоэмоциональное и психофизическое состояние пациентов, проводился опрос пациентов по вопросам организации стоматологической помощи, с каждым пациентом проводилось индивидуальное собеседование с привлечением студентов кафедры клинической психологии и психофизиологии УрФУ.

Всем пациентам перед операцией удаления зуба проводился контроль артериального давления, предлагалось проверить уровень сахара в крови экспресс-методами, антисептическая обработка полости рта 0,05\% раствором хлоргексидина биглюконата. Операция удаления зуба проводилась по традиционной методике.

Проведен анализ полученных эмпирических данных и обработка с помощью методов статистического анализа.

\section{Полученные данные и их обсуждение}

По данным проведенного исследования установлено, что 98,5\% всех испытуемых испытывают эмоциональное напряжение перед стоматологическим вмешательством. В контрольной группе (без эндокринной патологии) страх (выраженное эмоциональное напряжение с психофизиологическими проявлениями) перед стоматологическим вмешательством испытывали $61 \%$ пациентов, в группе пациентов, больных сахарным диабетом I типа, - 92\% человек, в группе с сахарным диабетом II типа - 79\%.

$39 \%$ всех испытуемых после стоматологического вмешательства отметили, что ожидание боли или манипуляции стоматолога, вызывающие незначительные болевые ощущения, ведут к возникновению эмоционального стресса, порой к неадекватному поведению и недоверию к действиям и рекомендациям врача стоматолога. Следствием этого чаще всего становится отказ от стоматологического лечения и, как следствие, прогрессирование существующих стоматологических заболеваний, возникновение новых, снижение качества жизни пациента, страдающего сахарным диабетом.

Проведенный опрос среди пациентов, посетивших нашу стоматологическую поликлинику, связанный с вопросами организации стоматологической помощи в контрольной группе, не выявил значимых проблем. При анкетировании пациентов с эндокринной патологией мы обратили особое внимание на замечания по вопросам организации приема больных, страдающих сахарным диабетом, проведены мероприятия организационного характера для создания комфортных условий записи на прием и получения стоматологической помощи в определенные часы для данной категории больных.

Проведенное нами исследование показывает, что стоматологическое вмешательство у больных сахарным диабетом чаще всего проходит со значительными колебаниями уровня глюкозы в крови в результате выброса в кровь адреналина и других адреналиноподобных веществ. Что ведет к негативным особенностям заживления операционной раны.

По нашим данным, у пациентов контрольной группы осложнения составили $0,05 \%$, сроки заживления лунки зуба соответствуют средним срокам заживления и составляли от 10 до 14 дней.

У пациентов с сахарным диабетом I типа лунки заживали в $6 \%$ случаев с осложнениями: $4 \%$ - по типу альвеолита, $1 \%$ - постинъекционная гематома в слизистую оболочку полости рта, 1\% - ограниченный остеомиелит лунки. Средние сроки заживления лунки у больных с сахарным диабетом I типа составили до двух месяцев.

Пациенты с сахарным диабетом II типа - 4\% послеоперационных осложнений. Из них: 3\% - альвеолит, $1 \%$ - ограниченный остеомиелит лунки. Средние сроки заживления лунок у пациентов с сахарным диабетом II типа в 11 \% случаев составили 1,5 месяца.

В послеоперационном периоде пациентам с сахарным диабетом I и II типа назначалась антибактериальная, противовоспалительная, десенсибилизирующая, общеукрепляющая терапия по показаниям. Проводилось обучение правилам индивидуальной гигиены полости рта, соблюдение диетотерапии, лицам с вредными привычками рекомендовался отказ от табакокурения.

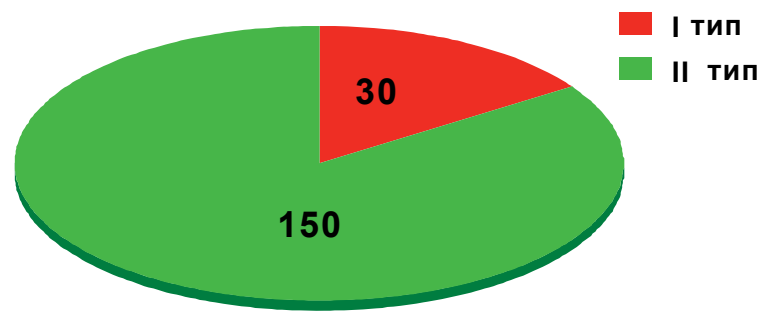

Количество пациентов с сахарным диабетом в зависимости от типа 
При проведении операции удаления зуба у пациентов с сахарным диабетом II типа для врача стоматолога-хирурга нами был определен следующий алгоритм:

1) вводилось минимальное количество анестетика;

2) использовались карпульные анестетики без вазоконстриктора или с минимальным количеством вазоконстриктора;

3) проводился контроль формирования кровяного сгустка в лунке удаленного зуба;

4) наложение швов на лунку удаленного зуба;

5) атравматичное удаление зуба, сглаживание острых краев альвеолы после удаления зуба;

6) контроль уровня сахара в крови до и после операции удаления зуба.

Учитывая данные проведенного нами исследования пациентов с сахарным диабетом I и II типа, определены организационные особенности приема пациентов с сахарным диабетом на хирургическом приеме:

1) прием пациентов с сахарным диабетом проводить в назначенное время, учитывая состояние уровня гликемии;

2) при оказании стоматологической помощи необходимо осуществлять тщательный сбор анамнеза у каждого пациента с целью выявления наличия сахарного диабета, уточнять тип диабета и выяснять продолжительность заболевания. Следует осторожно и предупредительно отнестись к пациенту, длительно болеющему диабетом, так как возможно возникновение конфликтных ситуаций (жалобы, неадекватное поведение на приеме). Особое значение обращалось нами на первое посещение врача стоматолога, во время которого достигался адекватный психоэмоциональный контакт с пациентом;

3) при каждом визите пациента врач формирует условия приема, исключающие создание стрессовых ситуаций;

4) при оказании хирургической помощи на амбулаторном стоматологическом приеме пациентам с сахарным диабетом необходима премедикация;

5) при проведении длительных и травматичных стоматологических манипуляций, учитывая отрицательное влияние эмоционального стресса на физиологическое состояние больных сахарным диабетом, лечение проводилось в несколько этапов, каждый из которых согласовывался с пациентом и объем лечения определялся индивидуально.

\section{Результат и обсуждение}

Результаты проведенного нами исследования доказывают, что врач на стоматологическом приеме должен учитывать следующие психофизиологические аспекты пациентов с сахарным диабетом:

При стрессе у пациента с сахарным диабетом развивается повышенный уровень глюкозы по такому же принципу, как и у здорового человека, но проблемы начинаются позднее, когда опасность проходит, и должны начаться процессы выравнивания сахара в крови. В контрольной группе специальные компенсаторные реакции организма постепенно приводили обмен веществ в состояние, соответствующее норме, у пациентов с сахарным диабетом возможность бороться с таким значительным выбросом глюкозы в кровь снижена или отсутствует. Существующие механизмы нормализации обмена веществ или не срабатывают, или действуют, но в недостаточной мере.

83\% больных сахарным диабетом I типа свойственен меланхолический (слабый, малоподвижный, неустойчивый) тип нервной системы; пациенты испытывают высокий уровень стоматологической тревожности. Среди больных сахарным диабетом II типа холерики (лица с сильным, неуравновешенным типом нервной системы) составляют порядка 78\%, при ожидании стоматологического лечения они менее тревожны. Врач стоматолог должен знать, что при увеличении длительности заболевания диабетом уровень тревожности возрастает.

Пациенты с сахарным диабетом I типа имеют обостренную болевую чувствительность, а диабетом II типа - сниженную. Пороги болевой чувствительности при сахарном диабете I типа в 1,4-2 раза выше, чем при сахарном диабете II типа.

У пациентов с сахарным диабетом I типа распространенность и выраженность стоматологических заболеваний, по нашим данным, составила $100 \%$, у пациентов с сахарным диабетом II типа $-87 \%$.

\section{Заключение}

Таким образом, у пациентов с сахарным диабетом I типа возникающая абсолютная недостаточность инсулина приводит к серьезным нарушениям всех видов обмена (углеводного, жирового, белкового, электролитного). Высокий уровень сахара крови, «закисление» крови недоокисленными продуктами, гипоксия тканей влияет на заживление лунок, их сроки после операции удаления зуба. Удаление зубов у пациентов, которые постоянно принимают инсулин, необходимо проводить утром, сразу после введения препарата (иногда врач-эндокринолог может рекомендовать повысить дозу инсулина). До процедуры удаления пациент должен позавтракать. Для профилактики инфицирования лунки рекомендуется прополоскать полость рта антисептическим раствором.

Работая с пациентами, страдающими сахарным диабетом, необходимо учитывать тип сахарного диабета, влияющего на лабильность настроения. Наличие у пациентов раздражительности, мнительности, тревожности, подавленности, угнетенности, общей слабости, адинамии, повышенной утомляемости, вялости, сонливости, снижение работоспособности, эмоциональной неустойчивости, повышенной конфликтности, 
хронических, довольно выраженных личностных изменений, обусловленных заболеванием, - прямое показание для обращения к клиническому психологу и проведения психотерапевтической подготовки перед стоматологическим вмешательством.

Небольшие хирургические вмешательства у больных компенсированными формами заболевания не требуют необходимости в изменении обычного режима лечения. При подготовке к плановой операции большого объема необходимо добиться полной компенсации диабета. Лечение пациентов, больных сахарным диабетом, проводится при создании обстановки психологического комфорта, так как конфликтные и неблагоприятные психологические ситуации могут приводить к декомпенсации диабета. В такой ситуации может помочь и своевременное применение седативных средств. Лабильность психоэмоционального состояния больных необходимо обязательно учитывать врачам-стоматологам при вербальном контакте с ними во время сбора данных анамнеза жизни и болезни, составлении плана лечения болезни и в дальнейшем. При особых, травмирующих больного, психических ситуациях, после консультации с лечащим врачом можно назначить транквилизаторы. При организации приема необходимо предусмотреть возможность для устранения начальных явлений гипогликемии.

При легких психонегативных синдромах пациентам, страдающим сахарным диабетом, вмешательство клинического психолога не требуется, врач стоматолог-хирург, подготовленный должным образом, дает полную информацию, внятные объяснения и практическую поддержку. Негативные психоэмоциональные состояния напрямую связаны с физиологией, что доказано еще в 1963 году Egberg. Сотрудники, проверяя кровяное давление и частоту пульса пациентов перед наркозом, отметили, что физиологические показатели и психологическое состояние пациента были в норме тогда, когда перед оперативным вмешательством врач подробно объяснял особенности и безопасность предстоящей процедуры. Пациент, уверенный в компетентности врача и понимающий механизмы предстоящего вмешательства, гораздо спокойнее реагирует на все процедуры, нежели тот, который неясно представляет себе, что ему предстоит. Врач-стоматолог должен планировать прием пациентов, имеющих эндокринную патологию и психологические особенности, так, чтобы было время для предварительного общения с пациентом. В более тяжелых случаях заболевания необходимо подключать специалиста - клинического психолога и применять уже специфические методы психотерапии, например, терапию, нацеленную на решение проблем, когнитивно-бихевиоральную терапию или психотерапию, применяемую при нарушениях межличностных отношений, которую проводит клинический психолог.

\section{Выводы}

В результате проведенного нами исследования для врачей-стоматологов, оказывающих медицинскую помощь пациентам, страдающим сахарным диабетом, разработан алгоритм проведения медицинского вмешательства. Врачи стоматологи-хирурги при планировании стоматологического вмешательства должны учитывать не только объем стоматологического вмешательства, тип сахарного диабета, но и психологические особенности протекания общего заболевания, влияющие на лабильность настроения, психофизиологические проявления у данной группы пациентов. Проявление психологических факторов у пациентов, таких как раздражительность, мнительность, тревожность, подавленность, угнетенность, эмоциональная неустойчивость, повышенная конфликтность, и психофизиологические проявления в виде общей слабости, адинамии, повышенной утомляемости, вялости, сонливости, снижения работоспособности, хронических, довольно выраженных личностных изменений, обусловленных заболеванием, - прямое показание для обращения к клиническому психологу и проведения психотерапевтической подготовки перед стоматологическим вмешательством. Задачи, поставленные нами при проведении исследования, выполнены в полном объеме, цель исследования достигнута.

\section{Литература}

1. Балаболкин М.И., Клебанова Е. М., Креминская В.М.: Лечение сахарного диабета и его осложнений. Руководство для врачей. - М.:Медицина, 2005. - 512

2. Пародонтология: национальное руководство/ под ред. Дмитриевой Л.А. - М.: ГЭОТАР-Медиа, 2013. - 712 с.

3. Рабинович С.А., Московец О.Н., Зорян Е.В., Демина Н.А., Ефремов О.С. Особенности болевого реагирования пациентов с сахарным диабетом на этапах амбулаторного стоматологического приема // Клиническая стоматология. 2007, № 1. С. 62 - 65.

4. Самойлик, М.М. Стоматологический статус больных инсулиннезависимым сахарным диабетом и его коррекция: Автореф. дис. канд. мед. наук. М., 2004. - 20 с.

5. Антонова, К.В. Психические расстройства в клинике эндокринных заболеваний / К. В. Антонова //Русский медицинский журнал. - 2006. 一№26. - С. 1889-1905.

6. Захарчук, Т. А. Депрессивные состояния у больных сахарным диабетом: автореф. дис.... канд. мед. наук /Т. А. Захарчук. - М., 2007. - С. 127-134.

7. Дробижев, М. Ю. Отношение к лечению у больных сахарным диабетом. Влияние сопутствующих депрессивных и тревожнофобических расстройств / М. Ю. Дробижев // Проблемы эндокринологии. - 2002. - Т. 48, № 5. - С. $37-39$. 
8. Анциферов, М. Б. Сахарный диабет и депрессивные расстройства / М. Б. Анциферов, М. Ю. Дробижев // Русский медицинский журнал. - 2003. - Т. 11, № 27. - С. 1480.

9. Прядеин В.П., Чернавский А.Ф. Страх как предмет психологического исследования. Психологические, психофизиологические, возрастные и гендерные характеристики. Монография Издатель: LAPLAMBERTAcademicPublishingGmbH\&Co. KGHeinrich-Bocking-Str. 6-8 66121 Saarbrucken, Germany. 2012/ 241 c.

10. Чернавский А.Ф., Зарипова Э.М., Леонова О.М. Создание психоэмоциональной комфортности для пациента на приеме у врача стоматолога Биосовместимые материалы и новые технологии в стоматологии: сборник статей Международной конференции. 27-28 ноября. - Казань: Изд-во Казан. ун-та, 2014. - 270 с. С 212-218.

\section{References}

1. Balabolkin M.I., Klebanova E. M., Kreminskaya V.M. Lechenie sakharnogo diabeta i ego oslozhneniy. Rukovodstvo dlya vrachey (Treatment of diabetes mellitus and its complications. Guide for doctors), Moscow, Meditsina, 2005. 512 p.

2. Parodontologiya: natsionalnoe rukovodstvo (Periodontology: national guide), Moscow, GEOTAR-Media, 2013,712 p.

3. Rabinovich S.A., Moskovets O. N., Zoryan E. V., Demina N.A., Efremov O. S. Klinicheskaya stomatologiya - Clinical dentistry, 2007, no. 1, pp. 62- 65 .

4. Samoylik M.M. Stomatologicheskiy status bolnykh insulinnezavisimym sakharnym diabetom i ego korrektsiya (Dental status of patients with insulin-independent diabetes mellitus and its correction), author's abstract of PhD Thesis, 2004, Moscow, 20 p.

5. Antonova K. V. Russkiy meditsinsky zhurnal - Russian Medical Journal, 2006, no. 26, pp. 1889-1905.

6. Zakharchuk T.A. Depressivnye sostoyaniya u bolnykh sakharnym diabetom (Depressive states of patients with diabetes mellitus), author's abstract of PhD Thesis, Moscow, 2007, pp. 127-134.

7. Drobizhev M. Yu. Problemy endokrinologii - Problems of Endocrinology, 2002, vol. 48, no. 5, pp. 37-39.

8. Antsiferov M. B., Drobizhev M.Yu. Russkiy meditsinsky zhurnal - Russian Medical Journal, 2003, Vol. 11, no. 27 , pp. 1480.

9. Pryadein V.P., Chernavsky A.F. Strakh kak predmet psikhologicheskogo issledovaniya. Psykhologicheskie, Psykhfiziologicheskie, vozratnye i gendernye kharakteristiki (Fear as an object of psychological study. Psychological, psychophysiological, age and gender characteristics), Saarbrucken, Germany, Lambert Academic Publishing, 2012, 241 p.

10. Chernavsky A.F., Zaripova E.M., Leonova O.M. Materialy Mezhdunarodnoy konferentsii «Biosovmestimye materialy $i$ novye tekhnologii $v$ stomatologii» (Proceedings of the International conference «Biocompatible materials and new technologies in dentistry») Kazan, 2014, pp. 212-218.

\footnotetext{
Авторы:

Игорь Александрович ПЕТРОВ

к.м.н., главный врач муниципального автономного учреждения «Стоматологическая поликлиника № 12»,

Екатеринбург, Российская Федерация

stomat12@mail.ru

Александр Фридрихович ЧЕРНАВСКИЙ

к.пс.н., доцент кафедры клинической психологии и психофизиологии ИСПН ФГАОУ ВО «УрФУ имени первого

Президента России Б.Н. Ельична», Екатеринбург,

Российская Федерация

docent.alex@mail.ru

\section{Араик Александрович БАДАЛЯН}

заведуюший хирургическим отделением муницииального автономного учреждения "Стоматологическая поликлиника № 12», Екатеринбург, Российская Федерациия

stomat12@mail.ru

Михаил Нрьевич ОГНЕВ

врач-стоматолог-хирург хирургического отделения

муниципального автономного учреждения

«Стоматологическая поликлиника № 12», Екатеринбург,

Российская Федерачия

stomat12@mail.ru
}

Поступила 21.01.2017

Принята к печати 09.03.2017

\author{
Authors: \\ Igor A. PETROV \\ PhD in medicine, Head Physician, Municipal Autonomous \\ Institution «Dental clinic № 12», Ekaterinburg, Russia \\ stomat12@mail.ru \\ Aleksandr F. CHERNAVSKY (Corresponding author) \\ PhD in psychological sciences, Associate Professor, Department \\ of Clinical Psychology and Psychophysiology, Institute of Social \\ and Political Sciences, Ural Federal University, Ekaterinburg, \\ Russia \\ docent.alex@mail.ru \\ Araik A. BADALYAN \\ Head of the Department of Surgery, Municipal Autonomous \\ Institution «Dental clinic № 12», Ekaterinburg, Russia \\ stomat12@mail.ru \\ Mikhail Yu. Ognev \\ Dental Surgery Doctor, Department of Surgery, Municipal \\ Autonomous Institution «Dental clinic № 12», \\ Ekaterinburg, Russia \\ stomat12@mail.ru
}

